\title{
Identification of long-non coding RNA UCA1 as an oncogene in renal cell carcinoma
}

\author{
YIFAN LI ${ }^{1-3}$, TIANTIAN WANG ${ }^{1,2}$, YUCHI LI $^{3,4}$, DUQUN CHEN ${ }^{1-3}$, ZUHU YU $^{1-3}$, LU JIN $^{1-3}$, LIANGCHAO NI $^{1}$, \\ SHANGQI YANG ${ }^{1}$, XIANGMING MAO $^{1}$, YAOTING GUI $^{2}$ and YONGQING LAI ${ }^{1,2}$ \\ ${ }^{1}$ Department of Urology and ${ }^{2}$ The Guangdong and Shenzhen Key Laboratory of Male Reproductive Medicine and Genetics, \\ Peking University Shenzhen Hospital, Shenzhen, Guangdong 518036; ${ }^{3}$ Department of Urology, Anhui Medical University, \\ Hefei, Anhui 230032; ${ }^{4}$ Department of Urology, Shantou University Medical College, Shantou, Guangdong 515041, P.R. China
}

Received March 6, 2015; Accepted January 4, 2016

DOI: $10.3892 / \mathrm{mmr} .2016 .4894$

\begin{abstract}
Renal cell carcinoma (RCC) is the most common type of kidney cancer in adults, which is associated with poor prognosis and high recurrence. Long non-coding RNAs (IncRNAs) have been reported to be dysregulated in cancer and to be important in the regulation of carcinogenesis, thus suggesting that this class of molecules may be used as biomarkers in cancer. The lncRNA urothelial carcinoma associated 1 (UCA1) has been observed to be upregulated and to function as an oncogene in certain types of cancer; however, the role of UCA1 in RCC remains to be elucidated. The present study aimed to determine the expression and function of UCA1 in RCC. Quantitative polymerase chain reaction (qPCR) was used to determine the expression levels of UCA1 in 46 paired RCC and adjacent normal tissue samples. Furthermore, qPCR was used to determine the expression levels of UCA1 in four RCC cell lines compared with the human embryonic kidney $293 \mathrm{~T}$ cell line. The impact of UCA1 on cell migration, proliferation and apoptosis was investigated by wound scratch assay, MTT and flow cytometry, respectively. The results of the present study demonstrated that UCA1 expression levels were significantly increased in RCC tissues and cells, as compared with the controls. Ectopic expression and gene silencing of UCA1 in RCC cell lines exerted opposite effects on cellular proliferation, migration and apoptosis, and the results suggested that UCA1 may function as an oncogene in RCC. These results indicated that UCA1 may be considered as a promising biomarker for diagnosis, and a therapeutic target in RCC.
\end{abstract}

Correspondence to: Professor Yongqing Lai, Department of Urology, Peking University Shenzhen Hospital, 1120 Lianhua Road, Shenzhen, Guangdong 518036, P.R. China

E-mail: yqlord@163.com

Key words: long non-coding RNAs, urothelial carcinoma associated 1 , renal cell carcinoma, oncogene
Further research is required to elucidate the role and target genes of UCA1 in RCC.

\section{Introduction}

Renal cell carcinoma (RCC) is the most common type of kidney cancer in adults, accounting for $\sim 90 \%$ of all renal tumors and $3.9 \%$ of all cancer cases $(1,2)$. RCC is characterized by a lack of early-warning signs, protean clinical manifestations, and resistance to radiotherapy and chemotherapy (3). As a result, $25 \%$ of patients present with advanced disease when initially diagnosed with RCC. Furthermore, a third of patients who undergo resection of localized disease exhibit recurrence (4). The survival of patients with localized tumors who undergo radical nephrectomy is markedly improved compared with patients with regional and distant metastasis, thus demonstrating the importance of early detection and treatment (5). Therefore, the detection of novel biomarkers to aid early diagnosis is required.

Recently, numerous studies have highlighted the role of a group of long non-coding RNAs (lncRNAs) in carcinogenesis, and suggested that this class of molecules may be used as biomarkers in cancer (6-11). LncRNAs (length, $200 \mathrm{nt}$ to $\sim 100,000$ nt) constitute a novel class of mRNA-like transcripts with no protein-coding capacity (7). In recent years, an increasing amount of IncRNAs have been identified and observed to play crucial regulatory roles in various biological processes, including embryonic development and carcinogenesis (8), at transcriptional, post-transcriptional and translational levels (9). For example, HOX transcript antisense RNA and prostate cancer associated transcript 1 , the first identified IncRNAs, have been demonstrated to be critical in cancer due to their interaction with the polycomb repressive complex 2 , by which they regulate numerous genes $(10,11)$. However, few studies have investigated the involvement of lncRNAs in renal cancer progression.

The lncRNA urothelial carcinoma associated 1 (UCA1), which was originally identified in bladder transitional cell carcinoma (12), has been reported to have a potential role in the progression of bladder cancer (13-15). UCA1 has been reported to be upregulated in several tumor tissues, including bladder, colon, cervix, lung, breast, colorectal and brain 
cancer (13,16-19). Furthermore, UCA1 promotes cell proliferation and transformation (anchorage-independent growth), increases cell motility and invasion, induces drug resistance, and reduces prognosis in cancer $(13,16,19)$. However, the role of UCA1 in renal cancer remains to be elucidated. The aim of the present study was to determine the expression of UCA1 in renal tissues and cell lines, and the function of UCA1 with regards to the regulation of cell growth, migration and apoptosis in renal cancer. Further research is required to investigate the roles and target genes of UCA1.

\section{Materials and methods}

Tumor tissues and cell lines. RCC tissue and adjacent normal tissue samples were collected from 46 patients with RCC from Peking University Shenzhen Hospital (Shenzhen, China). The tumor tissue samples contained $80-90 \%$ cancer cells, and the paired adjacent normal tissue samples were obtained from $\geq 2 \mathrm{~cm}$ away from the tumor border and were negative for tumor cells, as detected by microscopy. All fresh tissue samples were immediately immersed in RNAlater (Qiagen $\mathrm{GmbH}$, Hilden, Germany) following surgical resection, frozen in liquid nitrogen and stored at $-80^{\circ} \mathrm{C}$ until further use. Informed consent was obtained from participants for the use of their tissues in the present study, and the study protocol was approved by the Ethics Committees of Peking University Shenzhen Hospital. The diagnosis of RCC was histopathologically confirmed and the data on all subjects were obtained from medical records, pathology reports and personal interviews. The clinicopathological information of the patients is presented in Table I. Stage classification was performed according to the 2010 American Joint Committee on Cancer staging system (20).

The human RCC cell lines: 786-O, ACHN, 769P and Caki-2 cells, were obtained from the American Type Culture Collection (Manassas, VA, USA). The human embryonic kidney 293T (HEK-293T) cell line was purchased from the Type Culture Collection of the Chinese Academy of Medical Sciences (Shanghai, China). BamHI and EcoRI restriction enzymes were purchased from Takara Bio, Inc., (Tokyo, Japan). All cell lines were cultured in Dulbecco's modified Eagle's medium (DMEM; Gibco; Thermo Fisher Scientific, Inc., Waltham, MA, USA) supplemented with $10 \%$ fetal bovine serum (FBS; Gibco; Thermo Fisher Scientific, Inc.), $1 \%$ antibiotics (100 units $/ \mathrm{ml}$ penicillin and $100 \mathrm{mg} / \mathrm{ml}$ streptomycin sulfates) and $1 \%$ glutamate (all Gibco; Thermo Fisher Scientific, Inc.) at $37^{\circ} \mathrm{C}$ in a humidified chamber containing $5 \% \mathrm{CO}_{2}$.

RNA extraction, reverse transcription and reverse transcription-quantitative polymerase chain reaction ( $R T$-qPCR). Samples were homogenized using a motor-driven tissue grinder (OSE-Y10; Tiangen Biotech Co., Ltd., Beijing, China) prior to RNA extraction. Total RNA was extracted from the tissues (50-100 mg) and cell lines $\left(1.5 \times 10^{6}\right)$ using RNAiso Plus reagent (Takara Bio, Inc.) for $5 \mathrm{~min}$ at room temperature, according to the manufacturer's protocol. Following vigorous shaking by hand for $15 \mathrm{sec}$, the samples were centrifuged at 12,000 x g for $15 \mathrm{~min}$ at $4^{\circ} \mathrm{C}$. The concentration and quality of RNA was measured using the NanoDrop 2000/2000c spectrophotometer
Table I. Clinicopathological features of patients with renal cell carcinoma.

\begin{tabular}{lc} 
Characteristic & Number of ca \\
\hline Mean age range (years) & $51(27-72)$ \\
Gender & $29 / 17$ \\
Male/female & \\
Histological type & $41 / 5$ \\
Clear cell/papillary & \\
pT-stage & $25 / 19 / 2$ \\
T1/T2/T3+T4 & \\
Fuhrman grade & $15 / 20 / 8 / 3$ \\
I/II/III/IV & \\
AJCC clinical stages & $26 / 17 / 3$ \\
I/II/III+IV &
\end{tabular}

pT, primary tumor; AJCC, American Joint Committee on Cancer.

(Thermo Fisher Scientific, Inc., Pittsburgh, PA, USA) at 260 and $280 \mathrm{~nm}$ (A260/280 ratio). The RNA samples with 260/280 ratios 1.8-2.0 were used for further experiments. Total RNA was converted into cDNA using the PrimeScript ${ }^{\mathrm{TM}} \mathrm{RT}$ reagent kit (Takara Bio, Inc.) at $37^{\circ} \mathrm{C}$ for $15 \mathrm{~min}$ followed by $85^{\circ} \mathrm{C}$ for $5 \mathrm{sec}$ and was held at $4^{\circ} \mathrm{C}$.

The expression levels of UCA1 in tissues and cell lines were quantified using SYBR ${ }^{\circledR}$ Premix Ex Taq II (Takara Bio, Inc.), according to the manufacturer's protocol, on the Roche LightCycler $^{\circledR} 480$ Real-Time PCR system (Roche Diagnostics, Basel,Switzerland).PCR cycling conditions were as follows: $95^{\circ} \mathrm{C}$ for $15 \mathrm{~min}$, followed by 40 cycles at $95^{\circ} \mathrm{C}$ for $15 \mathrm{sec}$ and $55^{\circ} \mathrm{C}$ for $30 \mathrm{sec}$, and final extension at $72^{\circ} \mathrm{C}$ for $30 \mathrm{sec}$. Specific UCA1 and glyceraldehyde 3-phosphate dehydrogenase (GAPDH) primers were purchased from Invitrogen (Thermo Fisher Scientific, Inc.) (Table II). GAPDH was used as the endogenous control to normalize the data. The expression levels were presented as fold differences relative to GAPDH, based on the following equation: Relative quantification $=2^{-\Delta \Delta \mathrm{Cq}}\left[\Delta \Delta \mathrm{Cq}=\left(\right.\right.$ mean $\mathrm{Cq}_{\text {tumor }}-$ meanC $\mathrm{q}_{\text {control }}$ ) - (mean $\mathrm{Cq}_{\text {normal }}$ - mean $\mathrm{Cq}_{\text {control }}$ ) (21). RT-qPCR was performed in triplicate, including no-template controls. The amplification of the appropriate product was confirmed by melting curve analysis following amplification.

Ectopic expression and gene silencing of UCA1 in cells. For the analysis of overexpression, PCR-amplified UCA1, digested with BamHI and EcoRI restriction enzymes, was subcloned into the pcDNA3.1 mammalian expression vector (Invitrogen; Thermo Fisher Scientific, Inc.). Primers for the amplification of the full-length UCA1 were as follows: forward 5'-CCGGAA TTCTGACATTCTTCTGGACAATG-3' and reverse 5'-CCG CTCGAGCTGACTCTTTTAGGAAGATTTCT-3' (22). The UCA1 expression vector, pcDNA3.1-UCA1, was verified by sequencing. For the knockdown study of UCA1, two small interfering (si)RNAs targeting UCA1 (si-UCA1a and si-UCA1b) were designed and purchased from Shanghai GenePharma Co., Ltd. (Shanghai, China). The negative control (siRNA-NC), positive control (siRNA-GAPDH) and fluorescein amidite-labeled 
Table II. Sequences of siRNAs and primers.

\begin{tabular}{ll}
\hline Name & \multicolumn{1}{c}{ Sequence, 5'-3' } \\
\hline UCA1 & F: TCGGCTTAGTGGCTGAAGA \\
& R: GGTCCATTGAGGCTGTAGAGT \\
FAPDH & F: GGAGCGAGATCCCTCCAAAAT \\
& R: GGCTGTTGTCATACTTCTCATGG \\
si-UCA1a & F: UAGGAUCUGCAAUCAGAACUATT \\
& R:UAGUUCUGAUUGCAGAUCCUATT \\
si-UCA1b & F: GCACCUUGUUAGCUACAUAAA \\
& R: UAUGUAGCUAACAAGGUGCCA \\
siRNA-NC & F: UUCUCCGAACGUGUCACGUTT \\
& R: ACGUGACACGUUCGGAGATT \\
siRNA-GAPDH & F: GUAUGACAACAGCCUCAAGTT \\
& R: CUUGAGGCUGUUGUCAUACTT \\
siRNA-NC-FAM & F: UAUGUAGCUAACAAGGUGCCA \\
& R: UUCUCCGAACGUGUCACGUTT
\end{tabular}

UCA1, urothelial carcinoma associated 1; GAPDH, glyceraldehyde 3-phosphate dehydrogenase; siRNA/si, small interfering RNA; NC, negative control; FAM, fluorescein amidite; F, forward; R, reverse.

siRNA-NC (siRNA-NC-FAM) used in the present study were designed and purchased from Shanghai GenePharma Co., Ltd. The sequences of these siRNAs are presented in Table II.

Subsequently, the cells were transfected with pcDNA3.1-UCA1/pcDNA3.1 empty vector (mock), si-UCA1a, si-UCA1b, siRNA-NC, siRNA-GAPDH or siRNA-NC-FAM using Lipofectamine ${ }^{\circledR} 2000$ (Invitrogen; Thermo Fisher Scientific, Inc.), which was mixed with Opti-MEM ${ }^{\circledR}$ I Reduced Serum Medium (Gibco; Thermo Fisher Scientific, Inc.) $24 \mathrm{~h}$ after plating. Fluorescence microscopy (Axio Imager 2; Carl Zeiss AG, Oberkochen, Germany) and qPCR were used to assess and quantify transfection efficiency.

Cell motility assay. A wound scratch assay and a Transwell migration assay were performed to investigate the motility of RCC cells in vitro. Approximately $3 \times 10^{5} 786-\mathrm{O}$ or ACHN cells were seeded per 12 -well dish and transfected after $24 \mathrm{~h}$ with 100 pmol of either the siRNAs or $1 \mu \mathrm{g}$ vectors, using Lipofectamine $^{\circledR} 2000$. A total of $6 \mathrm{~h}$ post-transfection, a sterile $200 \mu \mathrm{l}$ pipette tip was used to scrape a clear line through the cell layer. The cells were washed with phosphate-buffered saline (PBS) and cultured in serum-free DMEM. Images of the scratches were captured using a digital camera system (DMIRB; Leica Microsystems GmbH, Wetzlar, Germany), 0 and $18 \mathrm{~h}$ after the scratches were made. The experiments were performed in triplicate and repeated $\geq 3$ times.

For the Transwell migration assays, following transfection with 100 pmol siRNAs and $1 \mu \mathrm{g}$ vectors in 12-well plates for $24 \mathrm{~h}, 6 \times 10^{4} 786-\mathrm{O}$ and ACHN cells were harvested and plated into the upper chambers (24-well insert; pore size, $8 \mu \mathrm{m}$; Corning Incorporated, Corning, NY, USA) with $150 \mu \mathrm{l}$ serum-free DMEM. DMEM (500 $\mu \mathrm{l})$ supplemented with $10 \%$ FBS was added to the lower chambers. The cells were cultured in a humidified chamber containing $5 \% \mathrm{CO}_{2}$ at $37^{\circ} \mathrm{C}$ for 2 days, prior to being fixed with paraformaldehyde for $25 \mathrm{~min}$ and stained with $0.1 \%$ crystal violet for $1 \mathrm{~h}$. The chambers were washed with PBS three times and dried prior to images being captured with a digital camera system. The experiments were performed in triplicate and repeated $\geq 3$ times.

Cell proliferation assay. Cell proliferation assay was performed using 3-(4,5-dimethylthiazol-2-yl)-2,5-diphenyltetrazolium bromide (MTT; Sigma-Aldrich, St. Louis, MO, USA). Approximately $5 \times 10^{3} 786-\mathrm{O}$ and ACHN cells were plated into a 96-well plate and transfected with either 13.3 pmol siRNA or $125 \mathrm{ng}$ vectors per well. At $0,24,48$ or $72 \mathrm{~h}$ post-transfection, $20 \mu \mathrm{l}$ MTT was added into five wells and incubated for $6 \mathrm{~h}$. The MTT was subsequently discarded and $120 \mu \mathrm{l}$ dimethyl sulfoxide (Sigma-Aldrich) was added to dissolve the purple formazan crystals. Following agitation for $30 \mathrm{~min}$ at room temperature, the optical value of each well was measured using an enzyme-linked immunosorbent assay microplate reader (iMark 680; Bio-Rad Laboratories, Inc., Hercules, CA, USA) at a wavelength of $490 \mathrm{~nm}$ (reference wavelength, $630 \mathrm{~nm}$ ).

Cell apoptosis assay. The apoptotic rate of RCC cells was determined by flow cytometry $\left(\right.$ EPICS $^{\circledR}$ Xl-4, Beckman Coulter, Inc., Brea, CA, USA). Approximately $3 \times 10^{5} 786-\mathrm{O}$ or ACHN cells were plated in 6-well plates for $24 \mathrm{~h}$ and were then transfected with 200 pmol siRNAs (si-UCA1 or si-NC) and $2 \mu \mathrm{g}$ (pcDNA3.1-UCA1 or mock) vectors. At $48 \mathrm{~h}$ post-transfection, the cells in each well, including the floating cells, were pelleted by centrifugation at $200 \mathrm{x}$ g The cells were washed twice with $4^{\circ} \mathrm{C}$ PBS, resuspended in $100 \mu 11 \mathrm{X}$ binding buffer and stained with $5 \mu \mathrm{l}$ propidium iodide and $5 \mu \mathrm{l}$ Annexin V-fluorescein isothiocyanate (Invitrogen; Thermo Fisher Scientific, Inc.) for $15 \mathrm{~min}$ at room temperature. Within $1 \mathrm{~h}$, each cell sample was added and mixed with $400 \mu \mathrm{l} 1 \mathrm{X}$ binding buffer prior to flow cytometry. Each experiment was conducted at least three times.

Statistical analysis. Relative UCA1 expression data were presented as the mean \pm standard error of the mean (Fig. 1). All remaining data were expressed as the mean \pm standard deviation of three independent experiments. Statistical analyses were performed using SPSS 19.0 statistical software (IBM SPSS, Armonk, NY, USA). Statistical significance was determined using Student's t-test. A paired t-test was used to compare the expression levels of UCA1 in matched tumor and adjacent normal samples. $\mathrm{P}<0.05$ was considered to indicate a statistically significant difference.

\section{Results}

UCA1 is upregulated in RCC tissues and cell lines. To determine the mRNA expression levels of UCA1, RT-qPCR was performed on 46 paired RCC tissue and adjacent normal tissue samples. Relative expression levels of UCA1 $\left(\log _{2}\right.$; $\mathrm{T} / \mathrm{N}$ ) are presented in Fig. 1A. The expression levels of UCA1 were significantly higher in RCC tissues, as compared with in adjacent normal tissues $(\mathrm{P}<0.01)$, as demonstrated in Fig. $1 \mathrm{~B}$.

In addition, the present study analyzed the expression levels of UCA1 in four RCC cell lines (786-O, ACHN, 769P and Caki-2) and one normal kidney human embryonic kidney 

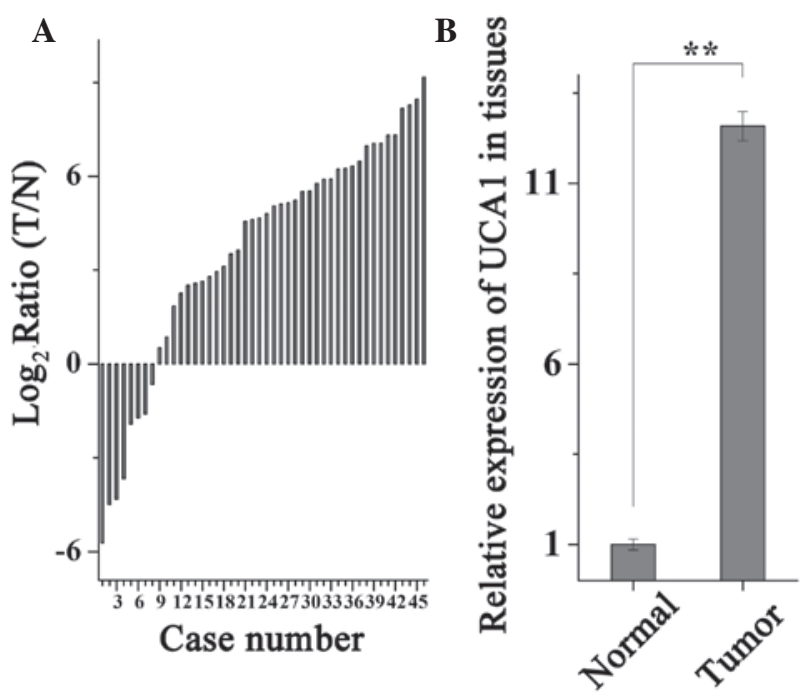

Figure 1. Expression of UCA1 in 46 paired RCC tissue and adjacent norma tissue samples. (A) $\log _{2}$ ratios of UCA1 expression in 46 paired RCC tissues (T) to normal kidney tissues (N). (B) Relative expression levels of UCA1 in RCC tumor tissue and normal tissue samples. Data are presented as the mean \pm standard error of the mean. ${ }^{* *} \mathrm{P}<0.01$. UCA1, urothelial carcinoma associated 1; RCC, renal cell carcinoma.

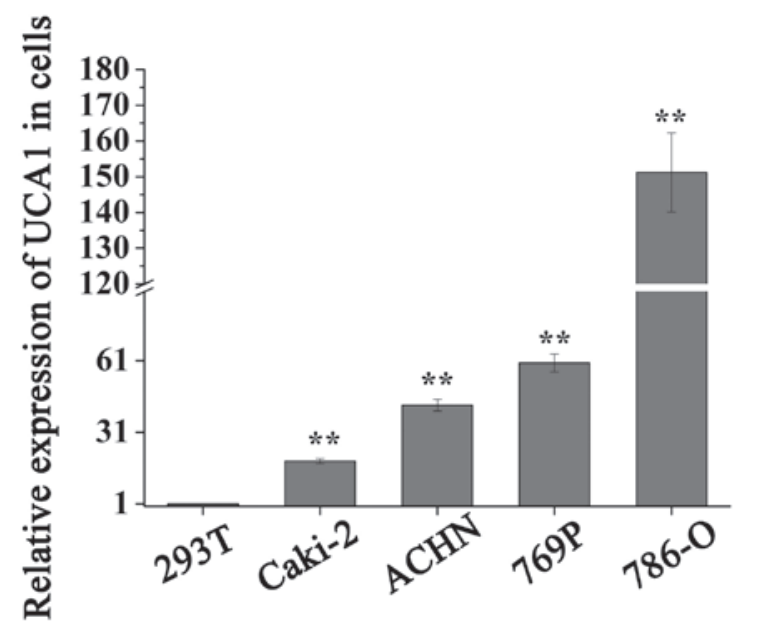

Figure 2. Expression of UCA1 in renal cell carcinoma cell lines (786-O, ACHN, 769P and Caki-2) and in normal human embryonic kidney 293T cells. Data are presented as the mean \pm standard deviation of three independent experiments. ${ }^{* *} \mathrm{P}<0.01$ vs. $293 \mathrm{~T}$ cells. UCA1, urothelial carcinoma associated 1 .

cell line (HEK-293T). As presented in Fig. 2, the expression levels of UCA1 were significantly higher in 786-O, 769P, ACHN and Caki-2 cells $(\mathrm{P}<0.01)$, as compared with the HEK-293T cells, which is consistent with the expression pattern of UCA1 in RCC tissues. In the functional experiments, two human RCC cell lines were selected. 786-O and 769P cells exhibited the highest expression levels of UCA1; however, ACHN exhibited more successful growth in the laboratory than 769P cells. Therefore, in the present study, 786-O and ACHN cells were selected for further experiments.

Validation of cell transfection efficiency. The transfection efficiency was $>90 \%$, when the cells were transfected with
FAM-conjugated siRNA-NC, as detected by fluorescence microscopy (Fig. 3A). The control experiment was performed by transfecting the cells with si-GAPDH and si-NC (Fig. 3B). As presented in Fig. 3C, to quantify the efficiency of the siRNA, qPCR was performed. The results indicated that UCA1 was significantly downregulated by 26.3 and $81.1 \%$ in ACHN cells $(\mathrm{P}<0.05)$, and by 15.8 and $83.4 \%$ in $786-\mathrm{O}$ cells $(\mathrm{P}<0.05)$ by si-UCA1a and si-UCA1b, respectively, as compared with the si-NC group. Therefore, knockdown of UCA1 expression was performed by transfecting the cells with si-UCA1b, in order to investigate the biological functions of UCA1 in RCC cells. To verify the overexpression efficiency, qPCR was performed following transfection with pcDNA3.1-UCA1 or a mock vector. UCA1 was significantly upregulated, 5.23 and 7.27 times compared with the mock group $(\mathrm{P}<0.01)$, in $\mathrm{ACHN}$ and $786-\mathrm{O}$ cells respectively (Fig. 3D).

UCA1 increases RCC cell motility. To investigate the effects of UCA1 on cell motility in 786-O and ACHN RCC cells, Transwell migration and cell scratch assays were conducted. As presented in Fig. 4A, the results of the Transwell migration assay demonstrated that the motility of cells transfected with si-UCA1b was significantly reduced by $30.5 \%(\mathrm{P}<0.01)$ in $786-\mathrm{O}$ cells and by $18.4 \%(\mathrm{P}<0.01)$ in ACHN cells, as compared with the si-NC group. Conversely, the motility of cells transfected with pcDNA3.1-UCA1 was significantly increased by $63.6 \%(\mathrm{P}<0.01)$ and $35.7 \%(\mathrm{P}<0.01)$ in $786-\mathrm{O}$ and ACHN cells, as compared with the mock group, respectively. Similar results were exhibited for the cell scratch assay. Downregulation of UCA1 by transfection of the cells with si-UCA1b significantly suppressed the migration of 786-O and ACHN cells by $34.5 \%(\mathrm{P}<0.01)$ and $39.0 \%(\mathrm{P}<0.01)$, as compared with the si-NC group. Conversely, upregulation of UCA1 by transfection with pcDNA3.1-UCA1 promoted migration by $23.1 \%(\mathrm{P}<0.01)$ and $34.5 \%(\mathrm{P}<0.05)$ in $786-\mathrm{O}$ and ACHN cells respectively, as compared with the mock group (Fig. 4B).

UCA1 promotes cell proliferation. A cell proliferation assay was performed using MTT in vitro. The results of the MTT assay suggested that upregulation of UCA1 promoted cell proliferation, whereas downregulation of UCA1 attenuated cell proliferation. As presented in Fig. 5A, the cell proliferation of 786-O and ACHN cells was significantly reduced by $10.31 \%(\mathrm{P}<0.05), 18.54 \%(\mathrm{P}<0.01)$ and $22.48 \%(\mathrm{P}<0.01)$, and $12.43 \%(\mathrm{P}<0.05), 14.62 \%(\mathrm{P}<0.01)$ and $16.70 \%(\mathrm{P}<0.01)$, following transfection with si-UCA1b for 24,48 and $72 \mathrm{~h}$ respectively, as compared with the si-NC group. Conversely, the proliferation of 786-O cells was significantly increased by $18.73 \%(\mathrm{P}<0.01), 20.94 \%(\mathrm{P}<0.01)$ and $22.98 \%(\mathrm{P}<0.01)$, and the proliferation of ACHN cells was significantly increased by $7.70 \%(\mathrm{P}<0.05), 13.91 \%(\mathrm{P}<0.01)$ and $25.87 \%(\mathrm{P}<0.01)$, at 24,48 and $72 \mathrm{~h}$ following transfection with pcDNA3.1-UCA1 respectively, as compared with the mock group (Fig. 5B).

UCA1 suppresses apoptosis. Apoptotic rate was quantified by flow cytometry (EPICS ${ }^{\oplus}$ Xl-4, Beckman Coulter, Inc.). UCA1 had a negative effect on apoptosis, indicating that ectopic expression of UCA1 suppressed apoptosis, whereas 

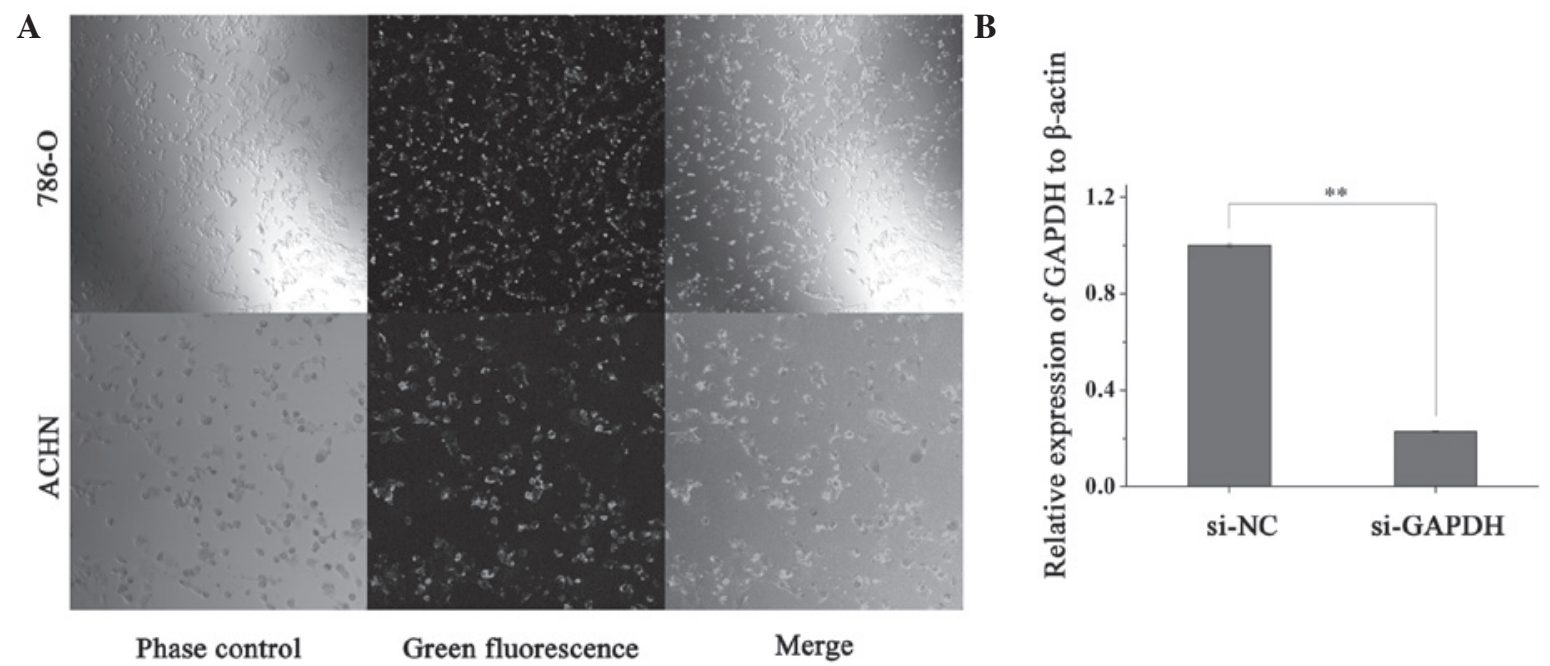

C

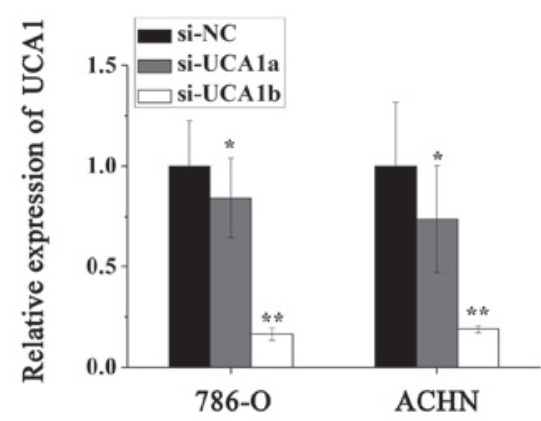

D

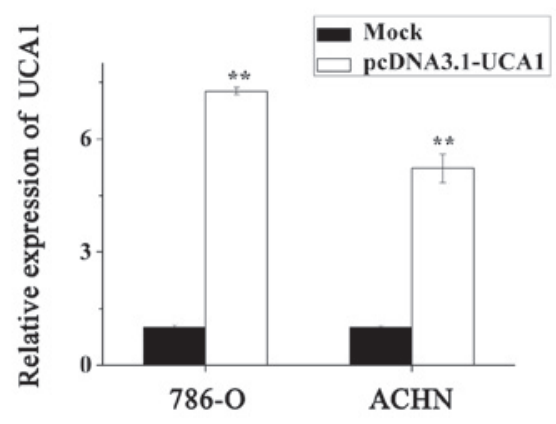

Figure 3. Validation of cell transfection efficiency. (A) Phase-contrast and green fluorescence images were captured in the same field (magnification, 500x) (B) Transfection with the positive control (si-GAPDH) was performed to determine the efficiency of the experiments. (C) Relative expression levels of UCA1 in 786-O and ACHN cells transfected with si-UCA1a, si-UCA1b or si-NC. (D) Relative expression levels of UCA1 in 786-O and ACHN cells transfected with pcDNA3.1-UCA1 or a mock vector. Data are presented as the mean \pm standard deviation of three independent experiments. ${ }^{*}<0.05$ and ${ }^{* *} \mathrm{P}<0.01$ vs. the si-NC/Mock group. UCA1, urothelial carcinoma associated 1; NC, negative control; si, small interfering RNA; GAPDH, glyceraldehyde 3-phosphate dehydrogenase.

downregulation of UCA1 increased apoptosis of RCC cells. Following transfection (48 h) with siRNAs (si-NC or si-UCAlb) or vectors (pcDNA3.1-UCA1 and mock), 786-O and ACHN cells were collected for apoptotic quantification. The early apoptotic rate of 786-O cells transfected with si-NC or si-UCA1b was 5.72 vs. $10.53 \%(\mathrm{P}<0.01)$ and the apoptotic rate of ACHN cells was 5.05 vs. $12.65 \%$ ( $\mathrm{P}<0.05$; Fig. 6A). As presented in Fig. 6B, upregulation of UCA1 by transfection with pcDNA3.1-UCA1 inhibited the apoptotic rate of 786-O and ACHN cells from 12.35 to $4.4 \%(\mathrm{P}<0.01)$ and from 11.27 to $2.45 \%(\mathrm{P}<0.01)$ respectively, as compared with mock transfection.

\section{Discussion}

RCC is a type of cancer associated with poor prognosis, since $\sim 25 \%$ of patients are initially diagnosed with advanced RCC, and up to $33.3 \%$ of patients with clinically localized disease develop recurrence post-surgery (4). It is therefore critical to identify novel biomarkers for the early diagnosis and monitoring of RCC, and as potential therapeutic targets for the treatment of RCC. LncRNAs have recently received attention and have been observed to be dysregulated in numerous types of disease, particularly in cancer (23). Dysregulated lncRNA expression is important in diverse cellular processes, including cell migration, invasion, proliferation and apoptosis $(7,24)$. Although several lncRNAs have been demonstrated as crucial in RCC, including onco-lncRNAs (metastasis associated lung adenocarcinoma transcript 1 and KCNQ1 opposite strand/antisense transcript 1) and tumor suppressor lncRNAs (growth arrest specific 5, H19 and maternally expressed 3) (25), the present study focused on the effects of UCA1 on the onset and development of RCC.

In the present study, the upregulation of UCA 1 in 46 paired RCC tissues and four RCC cell lines was compared with adjacent normal tissues and HEK-293T cells using qPCR. To investigate the function of UCA1 in RCC cell lines (786-O and $\mathrm{ACHN}$ ), primers were designed and constructed to confirm that si-UCA1b silenced the expression of UCA1 and that the overexpression vector pcDNA3.1-UCA1 upregulated the expression of UCA1. These models of up- and downregulation were then examined using various assays, including Transwell and scratch migration, MTT and apoptosis assays. The results demonstrated that suppression of UCA1 inhibited migration and cell proliferation, and induced cell apoptosis, whereas overexpression of UCA1 promoted migration and cell proliferation, and reduced cell apoptosis. These outcomes suggested that UCA1 may function as an oncogene in RCC by regulating cellular migration, proliferation and apoptosis.

Although aberrant UCA1 expression has been observed, and its function on the cell biology of migration, invasion, 
$\mathbf{A}$

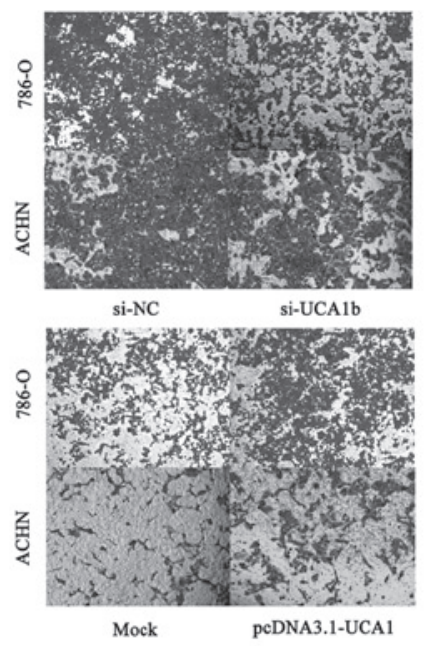

B
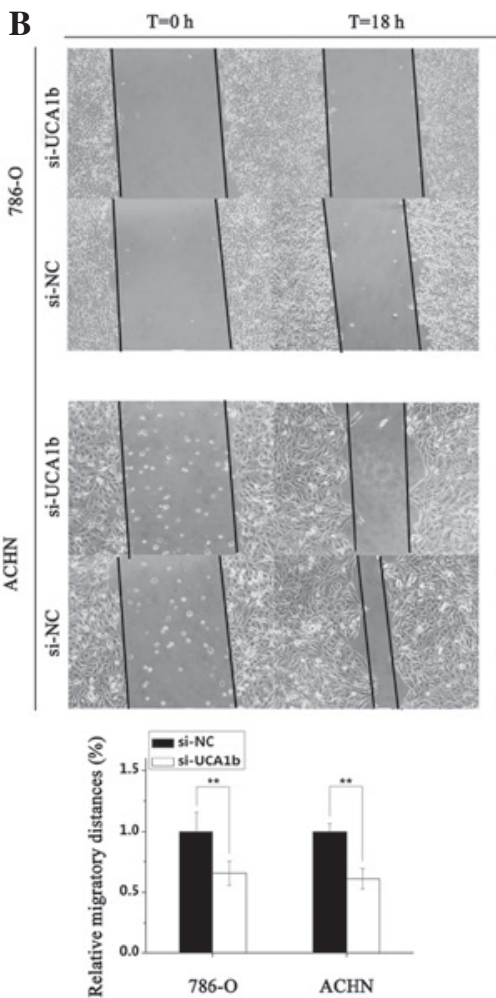

$\mathrm{T}=\mathbf{0} \mathrm{h}$
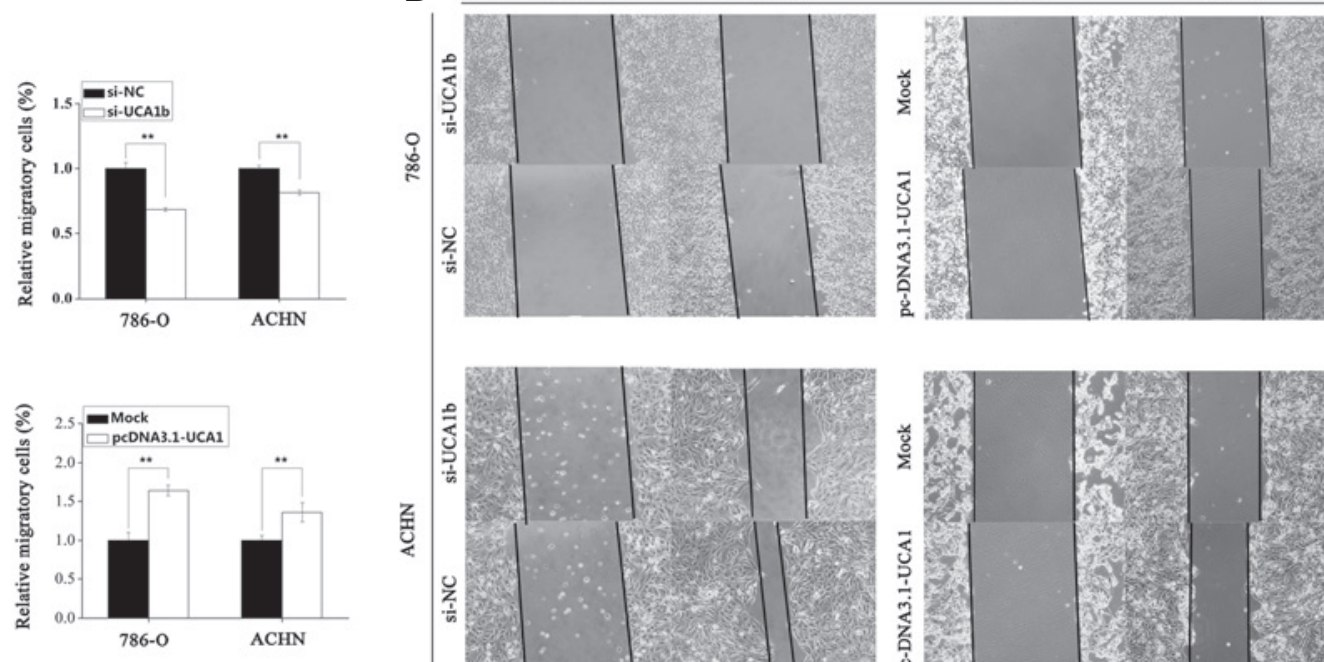
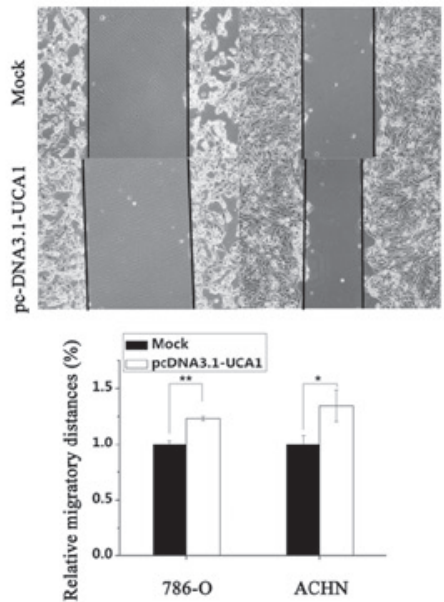

Figure 4. Cell motility assay. (A) Transwell migration assay (magnification, 400x) and (B) wound scratch assay (magnification, 200x) were used to examine the motility of 786-O and ACHN cells following transfection with si-UCA1b or si-NC, and pcDNA3.1-UCA1 or a mock vector. Data are presented as the mean \pm standard deviation of three independent experiments. ${ }^{*} \mathrm{P}<0.05,{ }^{* *} \mathrm{P}<0.01$. UCA1, urothelial carcinoma associated 1; NC, negative control; si, small interfering RNA.

A

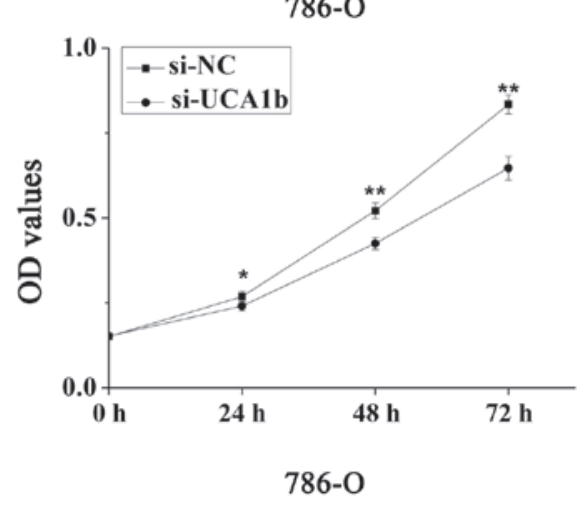

B

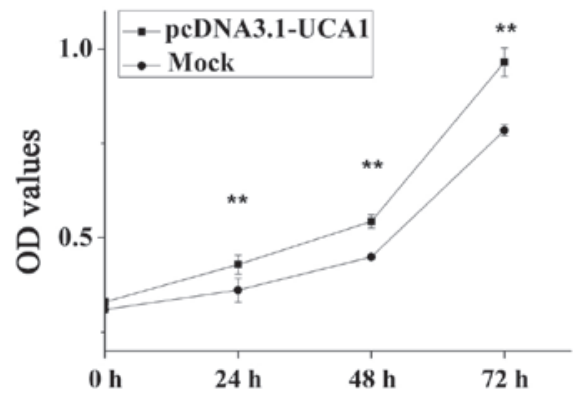

ACHN
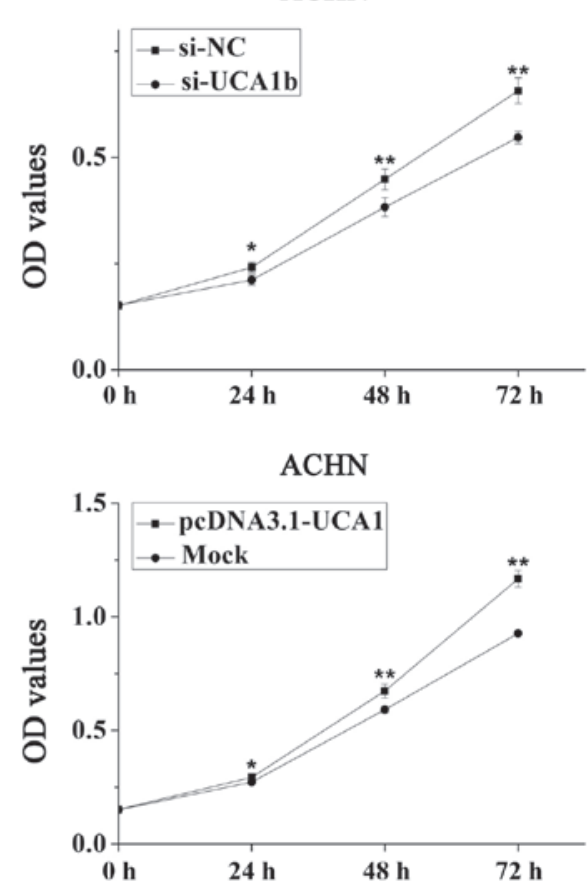

Figure 5. Cell proliferation of 786-O and ACHN cells transfected with (A) si-NC or si-UCA1b, and (B) pcDNA3.1-UCA1 or a mock vector was measured by 3-(4,5-dimethylthiazol-2-yl)-2,5-diphenyltetrazolium bromide assay. Data are presented as the mean \pm standard deviation of three independent experiments. ${ }^{*} \mathrm{P}<0.05$ and ${ }^{* *} \mathrm{P}<0.01$ vs. the si-NC/mock groups, . UCA1, urothelial carcinoma associated 1; OD, optical density; NC, negative control; si, small interfering RNA.

proliferation, apoptosis, glycolysis (26) and senescence (27) has been described in certain types of cancer, the signaling pathway involving UCA1 remains to be elucidated. LncRNAs function via chromatin modification, transcription initiation, 
A
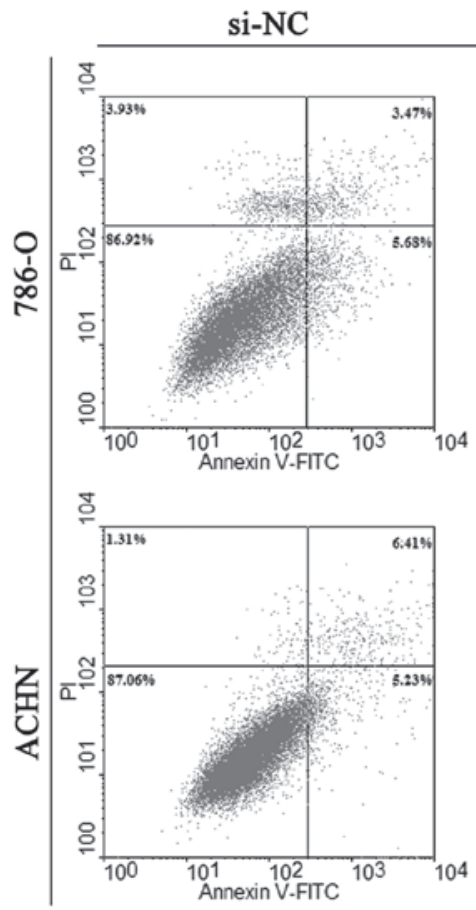

B
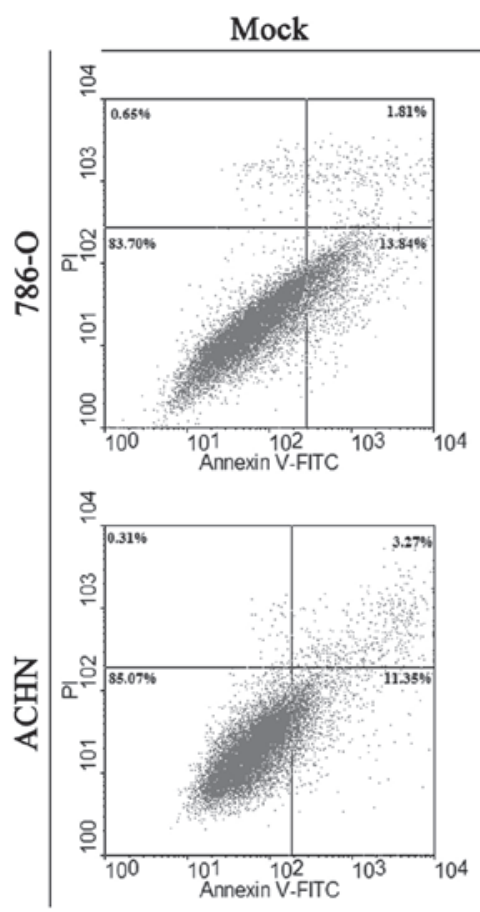

si-UCA1b

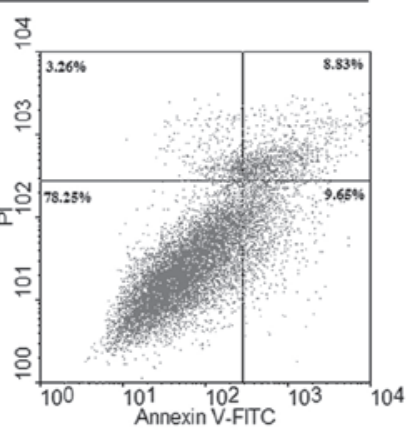

홍

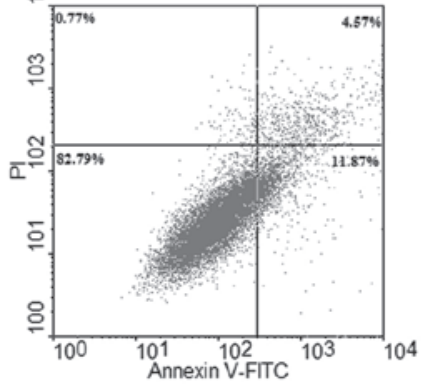

pcDNA3.1-UCA1

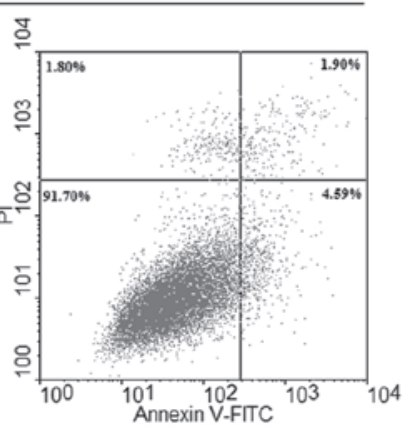

후

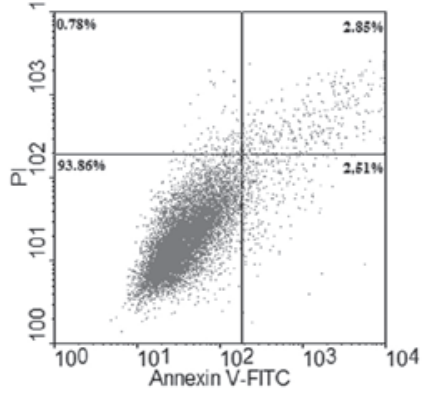

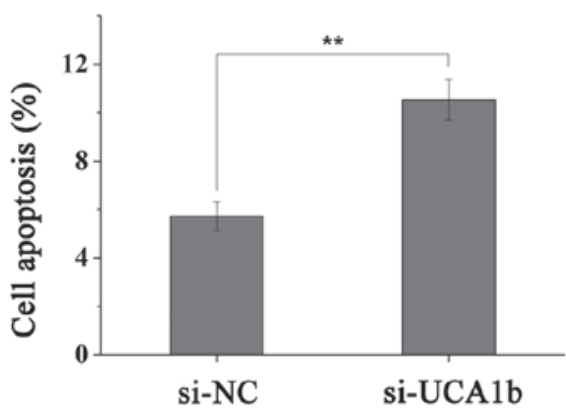
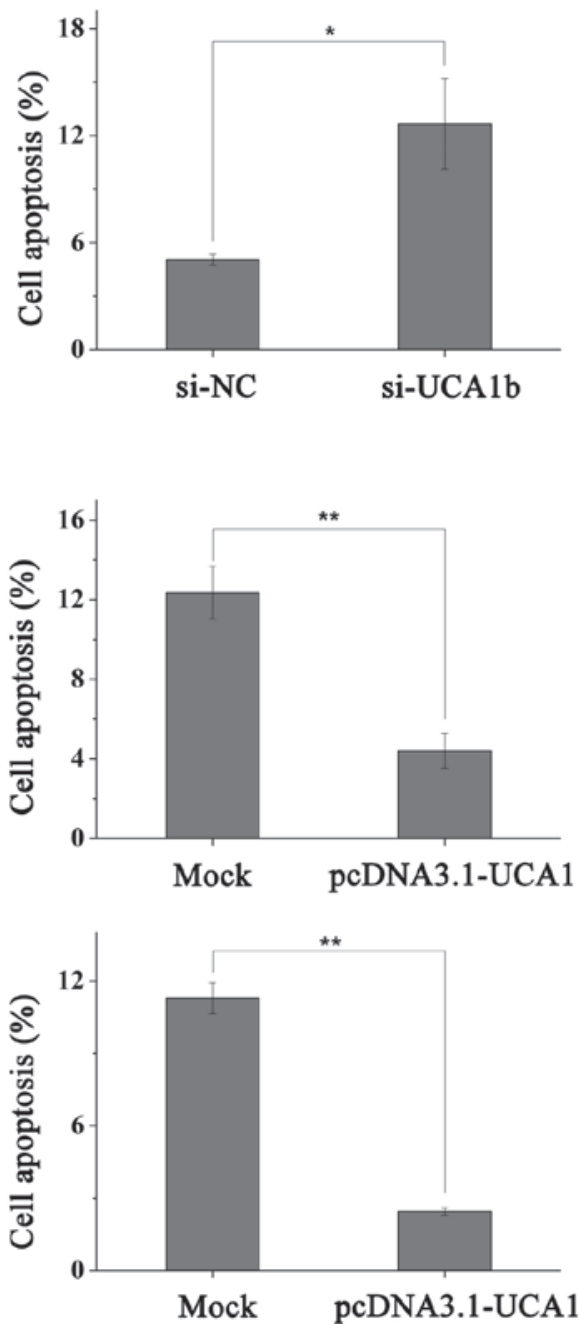

Figure 6. Rate of apoptosis (\%) of 786-O and ACHN cells transfected with (A) si-NC or si-UCA1b, and (B) pcDNA3.1-UCA1 or a mock vector was quantified by flow-cytometry. Data are presented as the mean \pm standard deviation of three independent experiments. ${ }^{*} \mathrm{P}<0.05$, ${ }^{* *} \mathrm{P}<0.01$. UCA1, urothelial carcinoma associated 1; NC, negative control; FITC, fluorescein isothiocyanate; PI, propidium iodide; si, small interfering RNA.

co- and post-transcriptional regulation or as competitive endogenous RNAs to microRNAs $(8,28)$. In bladder cancer, the UCA1 gene is localized in the cytoplasm and its expression is correlated with the progression of bladder cancer (14). In a microarray assay, UCA1a-overexpressing bladder cancer cells were shown to exhibit alterations in the gene expression of platelet-derived growth factor $\beta$ polypeptide, Fas and ATM serine/threonine kinase, which regulate cell apoptosis and tumorigenesis (29). Another study also applied microarray analysis and qPCR to identify and confirm the dysregulated genes affected by ectopic UCA1 expression, including upregulated wingless-type MMTV integration site family member 6, cytochrome P450, 1A1 and aurora kinase C, and downregulated methyl-CpG binding domain protein 3 and serine/arginine-rich protein-specific kinase 1 . These genes have already been detected in tumorigenesis, embryonic development and cisplatin resistance (13). However, the molecular mechanism remains to be elucidated and further studies are required to investigate how UCA1 affects these genes and exerts its function. 
In cancer development, UCA1 has been shown to be upregulated by transcription factor CCAAT/enhancer binding protein $\alpha$ (30) and hypoxia (31). In addition, overexpression of UCA1 may increase chemoresistance in bladder cancer by regulating Wnt signaling (32), and knockdown of UCA1 may accelerate cell cycle progression in bladder cancer via cAMP response element-binding protein and the phosphatidylinositol-4,5-bisphosphate 3-kinase dependent signaling pathway (15). UCA1 may also be involved in activation of the Akt signaling pathway by Ets-2, regulating apoptosis in bladder cancer cells (33). Aerobic glycolysis is critical for most cancer cells to generate the energy required for cellular processes, instead of the more efficient mitochondrial oxidative phosphorylation; this phenomenon is known as the Warburg effect (34). UCA1 promotes glycolysis by upregulating hexokinase 2 via activation of mammalian target of rapamycin-signal transducer and activator of transcription 3 and suppression of the microRNA-143 signaling pathway (26). The role of UCA1 and the UCA1-mediated signaling pathway in the onset and development of RCC remains to be elucidated; therefore, further studies are required to investigate and identify target genes of UCA1.

In clinical settings, UCA1 in urine may be used as a noninvasive biomarker, with a high level of sensitivity and specificity, for the early diagnosis and post-operative follow-up of transitional cell carcinoma $(12,35,36)$. In addition to bladder cancer, increased expression of UCA1 has been associated with the later stages and metastasis of melanoma (17). In colorectal cancer, a high level of UCA1 expression is notably correlated with a larger tumor size, less differentiated histology, greater tumor depth and a markedly poorer prognosis, as compared with in patients with low UCA1 expression (18).

In conclusion, UCA1 expression was upregulated in RCC tissues and cell lines. Due to the limited number of RCC samples used in the present study, no correlation was observed between the UCA1 expression and clinicopathological characteristics. Ectopic overexpression and gene silencing of UCA1 in RCC cell lines had opposite effects on cellular proliferation, migration and apoptosis, and the results suggested that UCA1 may function as an oncogene in RCC. The results of the present study indicated that UCA1 may be considered a promising biomarker for diagnosis and a potential therapeutic target in RCC. Further research is required to investigate the roles and target genes of UCA1.

\section{Acknowledgements}

The present study was supported by grants from the National Natural Science Foundation of China (grant no. 81101922), the Science and Technology Fund Project of Shenzhen (grant nos. JCYJ20130402114702124 and JCYJ20150403091443329) and the fund of Guangdong Key Medical Subject.

\section{References}

1. Tavani A and La Vecchia C: Epidemiology of renal-cell carcinoma. J Nephrol 10: 93-106, 1997.

2. National Cancer Institute: Surveillance, Epidemiology, and End Results Program. SEER stat fact sheets: Kidney and renal pelvis cancer. Available at: http://seer.cancer.gov/statfacts/html/kidrp. html. Accessed 30, 2014.
3. Motzer RJ, Bander NH and Nanus DM: Renal-cell carcinoma. N Engl J Med 335: 865-875, 1996.

4. Cohen HT and McGovern FJ: Renal-cell carcinoma. N Engl J 353: 2477-2490, 2005

5. Ridge CA, Pua BB and Madoff DC: Epidemiology and staging of renal cell carcinoma. Semin Intervent Radiol 31: 3-8, 2014.

6. Gibb EA, Brown CJ and Lam WL: The functional role of long non-coding RNA in human carcinomas. Mol Cancer 10: 38, 2011.

7. Ponting CP, Oliver PL and Reik W: Evolution and functions of long noncoding RNAs. Cell 136: 629-641, 2009.

8. Spizzo R, Almeida MI, Colombatti A and Calin GA: Long non-coding RNAs and cancer: A new frontier of translational research? Oncogene 31: 4577-4587, 2012.

9. Li X, Wu Z, Fu X and Han W: Long noncoding RNAs: Insights from biological features and functions to diseases. Med Res Rev 33: 517-553, 2013.

10. Takagi M, Absalon MJ, McLure KG and Kastan MB: Regulation of p53 translation and induction after DNA damage by ribosomal protein L26 and nucleolin. Cell 123: 49-63, 2005.

11. Prensner JR, Iyer MK, Balbin OA, Dhanasekaran SM, Cao Q, Brenner JC, Laxman B, Asangani IA, Grasso CS, Kominsky HD, et al: Transcriptome sequencing across a prostate cancer cohort identifies PCAT-1, an unannotated lincRNA implicated in disease progression. Nat Biotechnol 29: 742-749, 2011.

12. Wang XS, Zhang Z, Wang HC, Cai JL, Xu QW, Li MQ, Chen YC, Qian XP, Lu TJ, Yu LZ, et al: Rapid identification of UCA1 as a very sensitive and specific unique marker for human bladder carcinoma. Clin Cancer Res 12: 4851-4858, 2006.

13. Wang F, Li X, Xie X, Zhao L and Chen W: UCA1, a non-protein-coding RNA up-regulated in bladder carcinoma and embryo, influencing cell growth and promoting invasion. FEBS Lett 582: 1919-1927, 2008.

14. Xie XJ, Li X, Wang F and Chen W: Cellular localization and tissue expression pattern of UCA1, a non-coding RNA. Nan Fang Yi Ke Da Xue Xue Bao 30: 57-60, 2010 (In Chinese).

15. Yang C, Li X, Wang Y, Zhao L and Chen W: Long non-coding RNA UCA1 regulated cell cycle distribution via CREB through PI3-K dependent pathway in bladder carcinoma cells. Gene 496: 8-16, 2012.

16. Tsang WP, Wong TW, Cheung AH, Co CN and Kwok TT: Induction of drug resistance and transformation in human cancer cells by the noncoding RNA CUDR. RNA 13: 890-898, 2007.

17. Tian Y, Zhang X, Hao Y, Fang Z and He Y: Potential roles of abnormally expressed long noncoding RNA UCA1 and Malat-1 in metastasis of melanoma. Melanoma Res 24: 335-341, 2014.

18. Han Y, Yang YN, Yuan HH, Zhang TT, Sui H, Wei XL, Liu L, Huang P, Zhang WJ and Bai YX: UCA1, a long non-coding RNA up-regulated in colorectal cancer influences cell proliferation, apoptosis and cell cycle distribution. Pathology 46: 396-401, 2014.

19. Huang J, Zhou N, Watabe K, Lu Z, Wu F, Xu M and Mo YY: Long non-coding RNA UCA1 promotes breast tumor growth by suppression of p27 (Kip1). Cell Death Dis 5: e1008, 2014.

20. Edge SB, Byrd DR, Compton CC, Fritz AG, Greene FL, Trotti A (eds). AJCC cancer staging manual. 7th edition. Springer, New York, NY, pp 479-89, 2010.

21. Livak KJ and Schmittgen TD: Analysis of relative gene expression data using real-time quantitative PCR and the 2(-Delta Delta C(T)) Method. Methods 25: 402-408, 2001.

22. Wang T, Yuan J, Feng N, Li Y, Lin Z, Jiang Z, Gui Y: Hsa-miR-1 downregulates long non-coding RNA urothelial cancer associated 1 in bladder cancer. Tumour Biol 35: 10075-10084, 2014.

23. Shi X, Sun M, Liu H, Yao Y and Song Y: Long non-coding RNAs: A new frontier in the study of human diseases. Cancer Lett 339: 159-166, 2013.

24. Mercer TR, Dinger ME and Mattick JS: Long non-coding RNAs: Insights into functions. Nat Rev Genet 10: 155-159, 2009.

25. Martens-Uzunova ES, Böttcher R, Croce CM, Jenster G, Visakorpi T and Calin GA: Long noncoding RNA in prostate, bladder, and kidney cancer. Eur Urol 65: 1140-1151, 2014.

26. Li Z, Li X, Wu S, Xue M and Chen W: Long non-coding RNA UCA1 promotes glycolysis by upregulating hexokinase 2 through the mTOR-STAT3/microRNA143 pathway. Cancer Sci 105: 951-955, 2014.

27. Kumar PP, Emechebe U, Smith R, Franklin S, Moore B, Yandell M, Lessnick SL and Moon AM: Coordinated control of senescence by lncRNA and a novel T-box 3 co-repressor complex. Elife 3: e02805, 2014.

28. Yang L, Froberg JE and Lee JT: Long noncoding RNAs: Fresh perspectives into the RNA world. Trends Biochem Sci 39: 35-43, 2014. 
29. Wang Y, Chen W, Yang C, Wu W, Wu S, Qin X and Li X: Long non-coding RNA UCA1a(CUDR) promotes proliferation and tumorigenesis of bladder cancer. Int J Oncol 41: 276-284, 2012.

30. Xue M, Li X, Wu W, Zhang S, Wu S, Li Z and Chen W: Upregulation of long non-coding RNA urothelial carcinoma associated 1 by CCAAT/enhancer binding protein $\alpha$ contributes to bladder cancer cell growth and reduced apoptosis. Oncol Rep 31: 1993-2000, 2014

31. Xue M, Li X, Li Z and Chen W: Urothelial carcinoma associated is a hypoxia-inducible factor-1 $\alpha$-targeted long noncoding RNA that enhances hypoxic bladder cancer cell proliferation, migration, and invasion. Tumour Biol 35: 6901-6912, 2014.

32. Fan Y, Shen B, Tan M, Mu X, Qin Y, Zhang F and Liu Y: Long non-coding RNA UCA1 increases chemoresistance of bladder cancer cells by regulating Wnt signaling. FEBS J 281: 1750-1758, 2014.
33. Wu W, Zhang S, Li X, Xue M, Cao S and Chen W: Ets-2 regulates cell apoptosis via the Akt pathway, through the regulation of urothelial cancer associated 1 , a long non-coding RNA, in bladder cancer cells. PLoS One 82013, 2013.

34. Cairns RA, Harris IS and Mak TW: Regulation of cancer cell metabolism. Nat Rev Cancer 11: 85-95, 2011.

35. Srivastava AK, Singh PK, Rath SK, Dalela D, Goel MM and Bhatt ML: Appraisal of diagnostic ability of UCA1 as a biomarker of carcinoma of the urinary bladder. Tumour Biol 35: 11435-11442, 2014.

36. Zhang Z, Hao H, Zhang CJ, Yang XY, He Q and Lin J: Evaluation of novel gene UCA1 as a tumor biomarker for the detection of bladder cancer. Zhonghua Yi Xue Za Zhi 92: 384-387, 2012 (In Chinese). 